\title{
Structural basis for iron piracy by Neisserial lactoferrin binding protein B
}

\author{
$\underline{\text { Ravi Yadav }}{ }^{1}$, Srinivas Chakravarthy ${ }^{2}$, Courtney Daczkowski ${ }^{3}$, Nicholas Noinaj ${ }^{1,4}$ \\ ${ }^{1}$ Department of Biological Sciences, Purdue University (yadav15@purdue.edu) \\ ${ }^{2}$ BIO-CAT, Illinois Institute of Technology, Sector 18ID, Advanced Photon Source, Argonne \\ National Laboratory, Lemont, IL - 60439, USA (schakrav11@gmail.com) \\ 32Department of Biochemistry, Purdue University (daczkocm@gmail.com) \\ ${ }^{4}$ Purdue Institute for Inflammation, Immunology and Infectious Disease, Purdue University, West \\ Lafayette, IN - 47906, USA (nnoinai@purdue.edu)
}

Neisseria are exclusive human pathogens causing meningitis, septicemia and gonorrhea. During pathogenesis, Neisseria employ surface receptors to scavenge iron from iron-containing host proteins such as transferrin, lactoferrin, and hemoglobin. The lactoferrin binding protein (Lbp) system has been proposed to selectively hijack iron from lactoferrin in an effort to evade the host nutritional immunity. The Lbp system is composed of two components: a TonB-dependent transporter called lactoferrin binding protein $\mathrm{A}(\mathrm{LbpA})$, and a surface-exposed lipoprotein called lactoferrin binding protein $B(\mathrm{LbpB})$. In addition to iron-scavenging, LbpB provides protection against host antimicrobial peptides. The molecular mechanisms for exactly how the Lbp system functions remain unknown, largely due to the absence of structural and biochemical studies, which has also hindered the development of Lbp-based vaccines. In the current study, we used small-angle $X$-ray scattering and size-exclusion chromatography to show that LbpB forms a stable complex with lactoferrin in solution, and X-ray crystallography to determine the structure of LbpB in complex with lactoferrin at $2.85 \AA$. In our crystal structure of the complex, the N-lobe of LbpB interacts with the C-lobe of lactoferrin with a buried interface that is stabilized by extensive hydrogen bonding and salt-bridge networks. Structural alignments with known structures of LbpB$\mathrm{N}$-lobe and lactoferrin indicate virtually no conformational changes upon complex formation. Our structure also provides insight into LbpB's specificity towards holo-lactoferrin over apo-lactoferrin, which undergoes a significantly different conformation in the absence of iron which disrupts the extensive LbpB binding site. We propose that $\mathrm{LbpB}$ binding locks lactoferrin in an iron-bound state upon delivery to LbpA for iron extraction and import, which ensures efficient iron scavenging during Neisserial infections, much like that proposed for TbpB previously within the Tbp system. 